\title{
ON THE COMPETITIVE HARVESTING OF MARINE RESOURCES*
}

\author{
ALBERTO BRESSAN ${ }^{\dagger}$ AND VASILE STAICU ${ }^{\ddagger}$
}

\begin{abstract}
The paper is concerned with the optimal harvesting of a marine resource, described by an elliptic equation with Neumann boundary conditions and a nonlinear source term. We first consider a single agent, whose harvesting effort at various locations is described by a positive Radon measure. Necessary conditions for optimality are derived, complementing the existence result proved in [A. Bressan, G. Coclite, and W. Shen, SIAM J. Control Optim., 51 (2013), pp. 1186-1202]. The second part of the paper deals with a competitive scenario, where several groups of fishermen, from different coastal towns and hence with different cost functions, harvest the same marine resource. We prove the existence of a Nash equilibrium, which is characterized in terms of a suitable variational inequality.
\end{abstract}

Key words. optimal harvesting of a marine resource, necessary conditions for optimality, existence of Nash equilibrium, variational inequality, obstacle problem

AMS subject classifications. 35J25, 49K20, 49N90, 90C33, 91B76

DOI. $10.1137 / 18 \mathrm{M} 1192949$

1. Introduction. Mathematical models of fisheries have received much attention in recent literature [11, 15, 22, 23, 25]. Following [7], we consider here a harvesting problem in a multidimensional domain $\Omega$. The density of fish (or some other marine resource) is recovered as the solution $u$ to the semilinear elliptic equation with measure-valued coefficients

$$
\Delta u+f(x, u)-u \mu=0, \quad x \in \Omega,
$$

and Neumann boundary conditions

$$
\mathbf{n}(x) \cdot \nabla u(x)=0, \quad x \in \partial \Omega .
$$

By $\mathbf{n}(x)$ we denote the unit outer normal vector at the boundary point $x \in \partial \Omega$. The first term in (1.1) accounts for diffusion, while $f=f(x, u)$ yields the growth rate. The last term describes the decrease in the population due to harvesting. Here $\mu$ is a measure, describing the harvesting effort at various locations.

We remark that, by the nature of the problem, there is no reason to assume a priori that $\mu$ should be absolutely continuous w.r.t. Lebesgue measure. For example, $\mu$ may concentrate a positive mass along the boundary $\partial \Omega$. From a mathematical point of view, this makes the problem more challenging, since one has to rely on the theory of elliptic equations with measure-valued coefficients.

Throughout, we assume the following:

(A1) $\Omega \subset \mathbb{R}^{d}$ is a bounded, connected open set with $\mathcal{C}^{2}$ boundary.

* Received by the editors June 11, 2018; accepted for publication (in revised form) August 30, 2019; published electronically December 3, 2019.

https://doi.org/10.1137/18M1192949

Funding: This work was partially supported by Portuguese funds through CIDMA - Center for Research and Development in Mathematics and Applications, and the Portuguese Foundation for Science and Technology (FCT) within the project UID/MAT/04106/2013.

$\dagger$ Department of Mathematics, Penn State University, University Park, PA 16802 (bressan@math. psu.edu).

$\ddagger$ CIDMA and Department of Mathematics, University of Aveiro, Campus Universitário de Santiago, 3810-193 Aveiro, Portugal (vasile@ua.pt).

3961

Copyright $@$ by SIAM. Unauthorized reproduction of this article is prohibited. 
(A2) $f=f(x, u)$ is a $\mathcal{C}^{2}$ function such that, for some constants $M, K$, one has $(1.3)$ $f(x, 0) \geq 0, \quad f_{u}(x, 0)>0, \quad f_{u u}(x, u)<0, \quad f(x, M) \leq 0, \quad|f(x, u)| \leq K$ for all $x \in \bar{\Omega}$ and $u \in[0, M]$.

(A3) $\mu$ is a positive Radon measure, absolutely continuous w.r.t. capacity, supported on the compact set $\bar{\Omega}$.

Elliptic problems with measure data have been studied in several papers $[5,6,10$, $12]$ and are now fairly well understood. Following $[5,6]$ we denote by $\mathcal{M}_{0}$ the family of measures which vanish on Borel sets with zero capacity, so that

$$
\operatorname{cap}_{2}(V)=0 \quad \Longrightarrow \quad \mu(V)=0 .
$$

For the definition and basic properties of capacity, we refer to $[2,17]$.

Definition 1.1. Let $\mu$ be a measure in $\mathcal{M}_{0}$. A function $u \in \mathbf{L}^{\infty}(\Omega) \cap H^{1}(\Omega)$ is a solution to the elliptic problem (1.1)-(1.2) if

$$
-\int_{\Omega} \nabla u \cdot \nabla \phi d x+\int_{\Omega} f(x, u) \phi d x-\int_{\bar{\Omega}} u \phi d \mu=0
$$

for every test function $\phi \in \mathcal{C}_{c}^{\infty}\left(\mathbb{R}^{d}\right)$.

We recall that, for a function $u \in H^{1}(\Omega)$, the pointwise values of $u$ on the closure $\bar{\Omega}$ can be determined by the formula

$$
u(x)=\lim _{r \rightarrow 0+} \frac{1}{\operatorname{Vol}\left(B_{r}(x) \cap \Omega\right)} \int_{B_{r}(x) \cap \Omega} u(y) d y,
$$

where $B_{r}(x)$ denotes the open ball centered at $x$ with radius $r$. Since $\Omega$ is a domain with $\mathcal{C}^{2}$ boundary, this limit exists at all points $x \in \bar{\Omega}$ except possibly on a set of zero capacity $[17,18]$. Assuming that (1.4) holds, the last integral in (1.5) is thus meaningful.

We also observe that, by (1.3), if $u$ is a solution to (1.1), then the function $x \mapsto u(x)+K|x|^{2}$ is subharmonic. In turn (see [2]), this implies that the map $u$ is upper semicontinuous.

Example 1.2. Consider the open domain $\Omega=] 0,1[$, take $f(x, u)=1$, and let the measure $\mu$ be a single Dirac mass at the origin. Then the function $u(x)=1+x-\frac{x^{2}}{2}$ satisfies the requirements of Definition 1.1. Indeed, for every $\phi \in \mathcal{C}^{\infty}$ one has

$-\int_{\Omega} \nabla u \cdot \nabla \phi d x+\int_{\Omega} f(x, u) \phi d x-\int_{\bar{\Omega}} u \phi d \mu=-\int_{0}^{1}(1-x) \cdot \phi^{\prime} d x+\int_{0}^{1} \phi d x-\phi(0)=0$.

Notice that, since $\mu$ concentrates a positive mass at $0 \in \partial \Omega$, at the origin the boundary condition (1.2) is not satisfied in a classical sense. Indeed,

$$
\lim _{x \rightarrow 0+} u^{\prime}(x)=1 \neq 0 .
$$

However, the solution satisfies the balance equation

$$
\int_{\Omega} f d x-\int_{\bar{\Omega}} u d \mu=\int_{0}^{1} 1 d x-u(0)=0,
$$

showing that there is no flux across the boundary. 
To formulate an optimization problem, we consider, with an upper bound on the harvesting capacity,

$$
\mu(\bar{\Omega}) \leq \kappa_{0},
$$

together with a cost function $\gamma$ which satisfies the following:

(A4) $\gamma: \bar{\Omega} \mapsto \mathbb{R}_{+}$is a strictly positive $\mathcal{C}^{2}$ function. Namely,

$$
\gamma(x) \geq c_{0}>0 \quad \text { for all } x \in \bar{\Omega} .
$$

We think of $\gamma(x)$ as the cost for a harvesting at the point $x \in \bar{\Omega}$. In a realistic example, $\gamma(x)$ could depend on the distance to reach $x$ from the harbor where the fishing company is located. As in [7], we consider the following:

(OHP) Optimal Harvesting Problem.

$$
\text { maximize: } \quad \mathcal{J}(u, \mu) \doteq \int_{\bar{\Omega}}(u(x)-\gamma(x)) d \mu
$$

over all couples $(u, \mu)$, where $\mu \in \mathcal{M}_{0}$ is a positive measure on $\bar{\Omega}$ with total mass bounded by (1.7), and $u$ is a corresponding solution of (1.1).

By the analysis in [7], one has the following.

THEOREM 1.3. Under the assumptions (A1), (A2), and (A4), the optimal harvesting problem (OHP) has at least one optimal solution.

Indeed, the proof given in [7] covers more general cases where $f$ need not be concave w.r.t. $u$, and the cost function $\gamma$ is only assumed to be lower semicontinuous.

The purpose of the present paper is two-fold. In section 2 we derive necessary conditions satisfied by an optimal solution $\left(u^{*}, \mu^{*}\right)$ to the harvesting problem. The remaining three sections deal with a competitive scenario, where a large number of small fishing companies harvest the same marine resource.

We start by considering the case where all players share the same cost function $\gamma=\gamma(x)$. In this setting, an equilibrium solution is constructed by solving a family of variational inequalities, and establishing a suitable continuity property in terms of the parameter. In section 4 we study the case of finitely many groups of players with different costs $\gamma_{1}, \ldots, \gamma_{N}$. This model can describe several groups of fishermen based at different coastal towns $y_{1}, \ldots, y_{N}$. In this case, the cost $\gamma_{i}(x)$ will depend on the distance of the point $x$ from $y_{i}$. The existence of an equilibrium solution is here proved by a topological argument as in [8], writing the problem in the form of a variational inequality.

Finally, in section 5 we consider a continuum of small players, each with a different cost function $\gamma(x, \theta), \theta \in[0, \kappa]$. After introducing a definition of equilibrium solution, we prove the existence of such a solution within a class of generalized strategies.

We remark that, in all the above cases, the uniqueness of the equilibrium solution remains an interesting open problem for future investigation.

2. Necessary conditions for optimality. Consider the Optimal Harvesting Problem (OHP), assuming (A1), (A2), and (A4).

Let $\left(u^{*}, \mu\right)$ be an optimal pair. To derive necessary conditions, we first outline the main argument. Consider a family of perturbed measures of the form

$$
\mu_{\varepsilon}=\mu+\varepsilon \nu .
$$

Copyright $@$ by SIAM. Unauthorized reproduction of this article is prohibited. 
Let

$$
u_{\varepsilon}=u^{*}+\varepsilon v+o(\varepsilon)
$$

be the corresponding solution of

$$
\Delta u_{\varepsilon}+f\left(x, u_{\varepsilon}\right)-u_{\varepsilon} \mu_{\varepsilon}=0,
$$

with Neumann boundary conditions (1.2), always in the sense of Definition 1.1. A formal linearization procedure shows that the first order perturbation $v$ satisfies the linear, nonhomogeneous equation

$$
\left\{\begin{aligned}
\Delta v+f_{u}\left(x, u^{*}\right) v-v \mu & =u^{*} \nu, & & x \in \Omega, \\
\mathbf{n} \cdot \nabla v & =0, & & x \in \partial \Omega .
\end{aligned}\right.
$$

Differentiating the harvest functional w.r.t. $\varepsilon$, we obtain

$$
\left.\frac{d}{d \varepsilon} \mathcal{J}\left(u_{\varepsilon}, \mu_{\varepsilon}\right)\right|_{\varepsilon=0}=\int_{\bar{\Omega}}\left(u^{*}-\gamma\right) d \nu+\int_{\bar{\Omega}} v d \mu .
$$

Observe that (2.3) can be written as

$$
L v=u^{*} \nu
$$

where $L$ is a linear, self-adjoint operator. Hence, denoting by $\psi$ the solution to the linear problem

$$
L \psi=\mu,
$$

one has

$$
\langle v, \mu\rangle=\left\langle L^{-1} u^{*} \nu, \mu\right\rangle=\left\langle u^{*} \nu, L^{-1} \mu\right\rangle=\left\langle u^{*} \nu, \psi\right\rangle .
$$

We can thus write

$$
\left.\frac{d}{d \varepsilon} \mathcal{J}\left(u_{\varepsilon}, \mu_{\varepsilon}\right)\right|_{\varepsilon=0}=\int_{\bar{\Omega}}\left(u^{*}-\gamma\right) d \nu+\int_{\bar{\Omega}} \psi u^{*} d \nu,
$$

where $\psi \in H^{1}(\Omega)$ provides the solution to the linear, nonhomogeneous problem

$$
\left\{\begin{aligned}
\Delta \psi+f_{u}\left(x, u^{*}\right) \psi-\psi \mu & =\mu, & & x \in \Omega, \\
\nabla \psi \cdot \mathbf{n} & =0, & & x \in \partial \Omega .
\end{aligned}\right.
$$

In view of the above analysis, the necessary conditions for optimality can now be stated as follows.

THEOREM 2.1. Let the assumptions (A1), (A2), and (A4) hold, and let $\left(u^{*}, \mu\right) \in$ $H^{1}(\Omega) \times \mathcal{M}_{0}$ be an optimal pair. Call $\psi$ the solution to the adjoint linear problem (2.6). Then there exists a constant $p \geq 0$ such that

$$
\begin{cases}(1+\psi(x)) u^{*}(x)-\gamma(x) \leq p & \text { a.e. w.r.t. the Lebesgue measure } \mathcal{L}^{d} \text { on } \Omega, \\ (1+\psi(x)) u^{*}(x)-\gamma(x)=p & \text { a.e. w.r.t. the measure } \mu \text { on } \bar{\Omega}\end{cases}
$$

Proof. If $\mu$ is the zero measure, the conclusion of the theorem is straightforward. In the remainder of the proof we thus assume that $\mu$ is a bounded, nonzero Radon measure on $\bar{\Omega}$, absolutely continuous w.r.t. capacity. 
1. We start by showing that $u^{*}$ is uniformly positive on $\bar{\Omega}$. Indeed, by (1.8) one has $\gamma(x) \geq c_{0}>0$. Call $\chi_{\left\{u^{*} \leq \gamma\right\}}$ the characteristic function of the set

$$
\left\{x \in \bar{\Omega} ; u^{*}(x) \leq \gamma(x)\right\} .
$$

By the formula (1.6), since $\gamma \in \mathcal{C}^{2}$, the set (2.8) is measurable w.r.t. Lebesgue measure, and also w.r.t. the measure $\mu \in \mathcal{M}_{0}$.

Consider the reduced measure

$$
\tilde{\mu}=\chi_{\left\{u^{*} \geq \gamma\right\}} \cdot \mu .
$$

Since $\tilde{\mu} \leq \mu$, the system (1.1)-(1.2) has a solution $\tilde{u} \geq u^{*}$. If $\tilde{\mu} \neq \mu$, recalling (1.9) we have

$$
\mathcal{J}\left(u^{*}, \mu\right)<\mathcal{J}(\tilde{u}, \tilde{\mu})
$$

contradicting the optimality of $\left(u^{*}, \mu\right)$. We thus conclude

$$
u^{*}(x) \geq \gamma(x) \geq c_{0}
$$

for every point $x$ in the support of $\mu$.

Next, by the assumptions (1.3) we can choose another positive constant $\delta_{0}$, with $0<\delta_{0}<c_{0}$ such that

$$
f(x, u)>0 \quad \text { for all } x \in \bar{\Omega}, \quad 0<u \leq \delta_{0} .
$$

Consider the set

$$
\Omega_{0} \doteq\left\{x \in \Omega ; u^{*}(x)<\delta_{0}\right\},
$$

which is open because $u^{*}$ is upper semicontinuous. On this set, $u^{*}$ is a solution to

$$
\Delta u^{*}=-f\left(x, u^{*}(x)\right) \leq 0,
$$

with boundary conditions

$$
\mathbf{n}(x) \cdot \nabla u^{*}(x)=0, \quad x \in \partial \Omega_{0} \cap \partial \Omega .
$$

We now observe that the constant function $v(x)=\delta_{0}$ is a subsolution to (2.12), satisfying the Neumann conditions (2.13) together with

$$
v(x) \leq u^{*}(x), \quad x \in \partial \Omega_{0} \cap \Omega,
$$

on the remaining portion of the boundary $\partial \Omega_{0}$. A comparison argument now yields

$$
u^{*}(x) \geq v(x)=\delta_{0} \quad \text { for all } x \in \Omega_{0} .
$$

By the above definition of $\Omega_{0}$, this implies $u^{*}(x) \geq \delta_{0}$ for all $x \in \Omega$.

2. Following [10], it will be convenient to work in the Hilbert space $X_{\mu} \doteq H^{1}(\Omega) \cap$ $\mathbf{L}_{\mu}^{2}(\bar{\Omega})$, consisting of all functions $u \in H^{1}(\Omega)$ such that $u \in \mathbf{L}_{\mu}^{2}(\bar{\Omega})$. This definition makes sense because, as remarked in step 1, the pointwise values of any function $u \in H^{1}(\Omega)$ are uniquely determined up to a set of zero capacity.

On $X_{\mu}$ we shall use the norm generated by the inner product

$$
\langle u, v\rangle \doteq \int_{\Omega} \nabla u \cdot \nabla v d x+\int_{\bar{\Omega}} u v d \mu .
$$

Copyright $@$ by SIAM. Unauthorized reproduction of this article is prohibited. 
We claim that this is equivalent to the norm $\|u\|_{H^{1}(\Omega)}+\|u\|_{\mathbf{L}_{\mu}^{2}(\bar{\Omega})}$. Indeed, in the opposite case there would exist a sequence $u_{n} \in X_{\mu}$ such that

$$
\lim _{n \rightarrow \infty}\left(\int_{\Omega}\left|\nabla u_{n}(x)\right|^{2} d x+\int_{\bar{\Omega}}\left|u_{n}(x)\right|^{2} d \mu\right)=0, \quad\left\|u_{n}\right\|_{\mathbf{L}^{2}(\Omega)}=1 \quad \text { for all } n \geq 1 .
$$

Call $a_{n}$ the average value of $u_{n}$ on $\Omega$. Since $\Omega$ is bounded and connected, by Poincaré's inequality [16] there exists a constant $C$ such that

$$
\left\|u_{n}-a_{n}\right\|_{\mathbf{L}^{2}(\Omega)} \leq C\left\|\nabla u_{n}\right\|_{\mathbf{L}^{2}(\Omega)} \rightarrow 0 .
$$

By (2.15) and (2.16) one has the convergence $\left\|u_{n}-a_{n}\right\|_{H^{1}(\Omega)} \rightarrow 0$. Therefore,

$$
\int_{\Omega}\left|a_{n}\right|^{2} d \mu \leq \int_{\Omega}\left(\left|u_{n}(x)\right|^{2}+\left|u_{n}(x)-a_{n}\right|^{2}\right) d \mu \rightarrow 0 .
$$

Since $\mu$ is a positive nonzero measure, this implies $a_{n} \rightarrow 0$ and hence $\left\|u_{n}\right\|_{\mathbf{L}^{2}(\Omega)} \rightarrow 0$, contradicting the last condition in (2.15).

3. The fact that $X_{\mu}$ is complete can be proved as in [10]. The dual space of $X_{\mu}$ will be denoted by $X_{\mu}^{\prime}$. For every $\eta \in X_{\mu}^{\prime}$, by Riesz' theorem, there exists $w \in X_{\mu}$ such that

$$
\langle w, v\rangle \doteq \int_{\Omega} \nabla w \cdot \nabla v d x+\int_{\bar{\Omega}} w v d \mu=\eta(v) \quad \text { for all } v \in X_{\mu} .
$$

We denote by $\eta \mapsto \Lambda(\eta)$ this bounded linear operator from $X_{\mu}^{\prime}$ into $X_{\mu}$.

For the sake of convenience, we also introduce the function

$$
g(x, u) \doteq \frac{f(x, u)}{u} .
$$

By the properties of $f$ at (1.3) and (1.8), for $u \in\left[c_{0},+\infty\right.$ [, the function $u \mapsto g(x, u)$ is $\mathcal{C}^{2}$ positive, and strictly decreasing.

4. Next, consider any bounded Borel measurable functions $\varphi_{1}, \varphi_{2}: \Omega \mapsto \mathbb{R}$, with $\varphi_{1} \geq 0$. Set $\nu \doteq \varphi_{1} \mathcal{L}^{d}+\varphi_{2} \mu$, and define the 1-parameter family of measures

$$
\mu_{\varepsilon}=\mu+\varepsilon \nu=\mu+\varepsilon \varphi_{1} \mathcal{L}^{d}+\varepsilon \varphi_{2} \mu .
$$

We observe that the two maps

$$
u \mapsto g(x, u) u, \quad(u, \varepsilon) \mapsto \varepsilon\left(\varphi_{1} \mathcal{L}^{d}+\varepsilon \varphi_{2} \mu\right) u
$$

are both continuously differentiable (for $u$ close to $u^{*}$ ) as maps from $X_{\mu}$ into $X_{\mu}$. By a slight abuse of notation, we regard $g(x, u) u$ and $\varepsilon\left(\varphi_{1} \mathcal{L}^{d}+\varepsilon \varphi_{2} \mu\right) u$ also as elements of $X_{\mu}^{\prime}$, given by the maps

$$
v \mapsto \int_{\Omega} g(x, u) u(x) v(x) d x, \quad v \mapsto \int_{\Omega} \varepsilon u(x) v(x) \varphi_{1} d x+\int_{\bar{\Omega}} \varepsilon u v \varphi_{2} d \mu .
$$

5. Call $u_{\varepsilon}$ the solution to (2.2), taking $\mu_{\varepsilon}$ as in (2.18). Our next goal is to prove that the map $\varepsilon \mapsto u_{\varepsilon}$ is differentiable for $\varepsilon$ close to zero. This will be achieved by expressing $u_{\varepsilon}$ as the fixed point of a differentiable transformation, and using the implicit

Copyright $@$ by SIAM. Unauthorized reproduction of this article is prohibited. 
function theorem. Indeed, with the previous notation, according to Definition 1.1 one has

$$
u_{\varepsilon}=\Lambda\left(g\left(x, u_{\varepsilon}\right) u_{\varepsilon}\right)-\varepsilon \Lambda\left(\left(\varphi_{1} \mathcal{L}^{d}+\varphi_{2} \mu\right) u_{\varepsilon}\right) .
$$

Since $g \in \mathcal{C}^{2}$, the map

$$
(u, \varepsilon) \mapsto u-\Lambda(g(x, u) u)+\varepsilon \Lambda\left(\left(\varphi_{1} \mathcal{L}^{d}+\varphi_{2} \mu\right) u\right)
$$

is differentiable from $X_{\mu} \times \mathbb{R}$ into $X_{\mu}$. By the implicit function theorem [13, 14], to prove that at $\varepsilon=0$ the derivative (2.4) is well defined, it thus suffices to show that at $u=u^{*}$, the linear operator

$$
I-\Psi, \quad \Psi \doteq D_{u} \Lambda\left(g\left(x, u^{*}\right) u^{*}\right),
$$

has a bounded inverse. More precisely, $\Psi$ is the linear map

$$
v \mapsto \Psi(v) \doteq \Lambda\left(g_{u}\left(x, u^{*}\right) u^{*} v+g\left(x, u^{*}\right) v\right)
$$

from $X_{\mu}$ into itself.

6. Since $u^{*}$ is a uniformly positive, bounded solution to (1.1) and $g_{u}<0$, one has

$$
\Delta u^{*}-u^{*} \mu+\left[g_{u}\left(x, u^{*}\right) u^{*}\right] u^{*}+g\left(x, u^{*}\right) u^{*}=g_{u}\left(x, u^{*}\right)\left(u^{*}\right)^{2} \leq-2 \delta u^{*}
$$

for some $\delta>0$ and all $x \in \Omega$. By continuity, there exists $\varepsilon>0$ such that

$$
\Delta u^{*}-u^{*} \mu+\lambda^{-1}\left[g_{u}\left(x, u^{*}\right) u^{*}+g\left(x, u^{*}\right)\right] u^{*} \leq-\delta u^{*}
$$

for all $|\lambda-1| \leq \varepsilon$ and $x \in \Omega$.

We observe that $\Lambda$ is self-adjoint, hence the same is true of $\Psi$. To prove that $I-\Psi$ has a bounded inverse, we will show that any eigenvalue $\lambda$ of $\Psi$ must satisfy the inequality

$$
|\lambda-1|>\varepsilon .
$$

Assume that $\Psi(v)=\lambda v$ for some $v \in X_{\mu}$ and $\lambda>0$. According to Definition 1.1, this means

$$
\left\{\begin{aligned}
\Delta v-v \mu+\lambda^{-1}\left[g_{u}\left(x, u^{*}\right) u^{*}+g\left(x, u^{*}\right)\right] v & =0, & x \in \Omega, \\
\mathbf{n} \cdot \nabla v & =0, & x \in \partial \Omega .
\end{aligned}\right.
$$

Since $g, g_{u}$ are bounded and $\mu \geq 0$, it follows that $v$ is bounded.

Consider the linear semigroup generated by the parabolic equation

$$
\left\{\begin{array}{lll}
w_{t}=\Delta w-w \mu+\lambda^{-1}\left[g_{u}\left(x, u^{*}\right) u^{*}+g\left(x, u^{*}\right)\right] w, & & x \in \Omega, \\
\mathbf{n} \cdot \nabla w=0, & & x \in \partial \Omega .
\end{array}\right.
$$

If (2.23) fails, to reach a contradiction we argue as follows. On the one hand, the function $v$ in (2.24) is a time-invariant solution of (2.25). On the other hand, by (2.22) the function

$$
U(t, x)=e^{-\delta t} u^{*}(x)
$$


is an upper solution of (2.25), while $-U$ is a lower solution. Choosing $C$ such that

$$
-C u^{*}(x) \leq v(x) \leq C u^{*}(x),
$$

a comparison argument yields

$$
-C e^{-\delta t} u^{*}(x) \leq v(x) \leq C e^{-\delta t} u^{*}(x) .
$$

Hence $v=0$, achieving a contradiction.

The inequalities (2.27) follow from corresponding inequalities valid for the resolvent operators. For $\epsilon>0$ small, consider the linear operator $w \mapsto w_{\epsilon} \doteq E_{\epsilon}(w)$, defined by

$$
w_{\epsilon}=w+\epsilon\left(\Delta w_{\epsilon}-w_{\epsilon} \mu+\lambda^{-1}\left[g_{u}\left(x, u^{*}\right) u^{*}+g\left(x, u^{*}\right)\right] w_{\epsilon}\right),
$$

with Neumann boundary conditions on $\partial \Omega$. Notice that $E_{\epsilon}$ yields the backward Euler approximation of step $\epsilon$ for the linear parabolic equation (2.25). We also observe that $E_{\epsilon}$ is positive in the sense that

$$
w \geq 0 \quad \Longrightarrow \quad E_{\epsilon} w \geq 0 .
$$

We claim that

$$
v_{\epsilon} \doteq E_{\epsilon} v=v, \quad u_{\epsilon} \doteq E_{\epsilon} u^{*} \leq \frac{u^{*}}{1+\epsilon \delta}
$$

for all $\epsilon \geq 0$ small enough. Indeed, the first identity follows trivially from (2.24). On the other hand, setting $\tilde{u}=(1+\epsilon \delta)^{-1} u^{*}$, by (2.22) we obtain

$$
\begin{gathered}
\tilde{u}-\epsilon\left(\Delta \tilde{u}-\tilde{u} \mu+\lambda^{-1}\left[g_{u}\left(x, u^{*}\right) u^{*}+g\left(x, u^{*}\right)\right] \tilde{u}\right) \\
\geq(1+\epsilon \delta)^{-1} u^{*}+\epsilon \delta(1+\epsilon \delta)^{-1} u^{*}=u^{*}
\end{gathered}
$$

for all $\epsilon>0$ small enough. We can write $u_{\epsilon}=\tilde{u}+u^{\sharp}$, where

$$
u^{\sharp}=E_{\epsilon}\left(u^{*}-\tilde{u}+\epsilon\left(\Delta \tilde{u}-\tilde{u} \mu+\lambda^{-1}\left[g_{u}\left(x, u^{*}\right) u^{*}+g\left(x, u^{*}\right)\right] \tilde{u}\right)\right) .
$$

By linearity, (2.29) and (2.31) imply $u^{\sharp} \leq 0$. Hence $u_{\epsilon} \leq \tilde{u}$.

Since $u \geq v$ and the backward Euler operator $E_{\epsilon}$ preserves ordering, we have

$$
v=E_{\epsilon} v \leq E_{\epsilon} u \leq \tilde{u}=\frac{u^{*}}{1+\epsilon \delta} .
$$

Calling $u(t, \cdot)$ the solution of $(2.25)$ with initial data $u(0, \cdot)=u^{*}$, the representation formula

$$
u(t, \cdot)=\lim _{n \rightarrow \infty}\left(E_{t / n}\right)^{n}\left(u^{*}\right)
$$

together with (2.26) and (2.32) yields

$$
v(t, \cdot) \leq C \lim _{n \rightarrow \infty}\left(\frac{1}{1+t n^{-1} \delta}\right)^{n} u^{*}=C e^{-\delta t} u^{*} .
$$

This proves that all of the eigenvalues of the self-adjoint linear operator $\Psi$ are bounded away from 1 . Hence $I-\Psi$ has a bounded inverse, as claimed. We thus 
conclude that the derivative in (2.5) is well defined for $\varepsilon$ in a neighborhood of the origin.

7. Next, let $\varphi$ be any Borel measurable function on $\bar{\Omega}$ such that

$$
\|\varphi\|_{\mathbf{L}^{\infty}(\mu)}<1, \quad \int_{\bar{\Omega}} \varphi d \mu=0 .
$$

For $|\varepsilon| \leq 1$, consider the measures

$$
\mu_{\varepsilon} \doteq(1+\varepsilon \varphi) \mu .
$$

Note that each $\mu_{\varepsilon}$ is a nonnegative Radon measure which satisfies (1.7).

Let $u_{\varepsilon} \in H^{1}(\Omega)$ be the solution to (2.2) with Neumann boundary conditions (1.2), always in the sense of Definition 1.1. The optimality condition implies

$$
0=\left.\frac{d}{d \varepsilon} \mathcal{J}\left(u_{\varepsilon}, \mu_{\varepsilon}\right)\right|_{\varepsilon=0}=\int_{\bar{\Omega}}\left(v+u^{*} \varphi-\gamma \varphi\right) d \mu=\int_{\bar{\Omega}}\left[(\psi+1) u^{*}-\gamma\right] \varphi d \mu .
$$

Here the function $\psi$ is the solution to the adjoint problem (2.6). Indeed, this formula corresponds to (2.5), with $\nu=\varphi \mu$.

If the function $(\psi+1) u^{*}-\gamma$ does not coincide with a constant a.e. w.r.t. the measure $\mu$, we can find a function $\varphi \in \mathbf{L}_{\mu}^{\infty}(\bar{\Omega})$ satisfying (2.33) such that the righthand side of (2.35) is nonzero, attaining a contradiction.

For some constant $p$ we thus have

$$
(\psi(x)+1) u^{*}(x)-\gamma(x)=p,
$$

$\mu$-a.e. on the domain $\bar{\Omega}$. This proves the second statement in (2.7).

8. Finally, if the first statement in (2.7) fails, then there exists $\delta>0$ and a Borel set $V \subset \Omega$ with Lebesgue measure meas $(V)>0$ such that

$$
(1+\psi(x)) u^{*}(x)-\gamma(x) \geq p+\delta \quad \text { for all } x \in V .
$$

Choose a set $V^{\prime} \subset \bar{\Omega}$, disjoint from $V$, such that $\mu\left(V^{\prime}\right)>0$. Define the functions $\varphi_{1}, \varphi_{2}$ by setting

$\varphi_{1}(x)=\left\{\begin{array}{cl}{[\operatorname{meas}(V)]^{-1}} & \text { if } x \in V, \\ 0 & \text { if } x \notin V,\end{array} \quad \varphi_{2}(x)=\left\{\begin{array}{cl}{\left[\mu\left(V^{\prime}\right)\right]^{-1}} & \text { if } x \in V^{\prime}, \\ 0 & \text { if } x \notin V^{\prime} .\end{array}\right.\right.$

For all $\varepsilon>0$ small enough, the measure

$$
\mu_{\varepsilon} \doteq \mu+\varepsilon \varphi_{1} \mathcal{L}^{d}-\varepsilon \varphi_{2} \mu
$$

is admissible. Indeed, $\mu_{\varepsilon}(\bar{\Omega})=\mu(\bar{\Omega})$. Calling $u_{\varepsilon}$ the corresponding solution to (1.1) and using (2.35)-(2.37), we now compute

$$
\begin{aligned}
\left.\frac{d}{d \varepsilon} \mathcal{J}\left(u_{\varepsilon}, \mu_{\varepsilon}\right)\right|_{\varepsilon=0} & =\int_{\Omega}\left[(\psi+1) u^{*}-\gamma\right] \varphi_{1} d x-\int_{\bar{\Omega}}\left[(\psi+1) u^{*}-\gamma\right] \varphi_{2} d \mu \\
& \geq \int_{V}(p+\delta) \varphi_{1} d x-\int_{V^{\prime}} p \varphi_{2} d \mu=(p+\delta)-p=\delta>0 .
\end{aligned}
$$

This contradicts the optimality of the pair $\left(u^{*}, \mu\right)$, proving the first inequality in (2.7). 
3. A noncooperative game. Next, instead of a single fishing company, we consider a large number of small, competing companies. We denote by $\kappa>0$ the joint harvesting capability of these companies, while $\mu$ will describe the combined harvesting effort. In this setting, the concept of a Nash equilibrium solution can be introduced, where each player has the choice of fishing at some point $x$ or remaining idle.

DEFINITION 3.1 (Nash equilibrium with several small players having the same cost). Consider a harvesting game governed by (1.1)-(1.2). Let $\kappa>0$ be the total harvesting capability, and let $\gamma(x)$ be the cost of fishing at $x$.

We say that $(u, \mu)$ is an equilibrium solution to the harvesting game provided that:

- $\mu$ is a positive measure on $\bar{\Omega}$, with $0 \leq \mu(\bar{\Omega}) \leq \kappa$.

- $u$ is a strictly positive solution to the elliptic equation (1.1) with Neumann boundary conditions (1.2).

- There exists a constant $p \geq 0$ such that

$$
\begin{cases}u(x)-\gamma(x)=p & \text { for all } x \in \operatorname{Supp}(\mu), \\ u(x)-\gamma(x) \leq p & \text { for all } x \in \bar{\Omega} .\end{cases}
$$

Moreover, one has the implication

$$
p>0 \quad \Longrightarrow \quad \mu(\bar{\Omega})=\kappa .
$$

Remark 3.2. Here $u(x)-\gamma(x)$ is the payoff for a unit effort of fishing at the point $x$. Since we are assuming that $(u, \mu)$ represents a Nash equilibrium, this payoff must be constant over the support of $\mu$, and smaller at points outside $\operatorname{Supp}(\mu)$ where nobody is fishing.

If the total mass of $\mu$ equals $\kappa$, this means that all fishermen are actively working. On the other hand, if $\mu(\bar{\Omega})<\kappa$, some fishermen remain idle. This is possible precisely when the payoff satisfies $u(x)-\gamma(x) \leq 0$ for all $x \in \bar{\Omega}$. In this case, there is no advantage in working rather than remaining idle.

TheOREm 3.3. In the setting of Definition 3.1, let $\kappa>0$ be given, and assume that (A1), (A2), and (A4) hold. Then the harvesting game admits an equilibrium solution $(u, \mu)$.

Proof. An equilibrium solution will be constructed in several steps.

1. Fix $p \geq 0$. We first show the existence of a unique uniformly positive solution to the obstacle problem (see Figure 1):

$$
\begin{gathered}
u(x) \leq \gamma(x)+p, \\
\begin{cases}\Delta u+f(x, u) \geq 0 \quad \text { if } x \in \Omega, \\
\mathbf{n}(x) \cdot \nabla u(x) \leq 0 \quad \text { if } x \in \partial \Omega,\end{cases} \\
u(x)<\gamma(x)+p \quad \Longrightarrow \quad\left\{\begin{array}{l}
\Delta u+f(x, u)=0 \\
\mathbf{n}(x) \cdot \nabla u(x)=0
\end{array} \quad \text { if } x \in \Omega,\right.
\end{gathered}
$$

Copyright $@$ by SIAM. Unauthorized reproduction of this article is prohibited. 


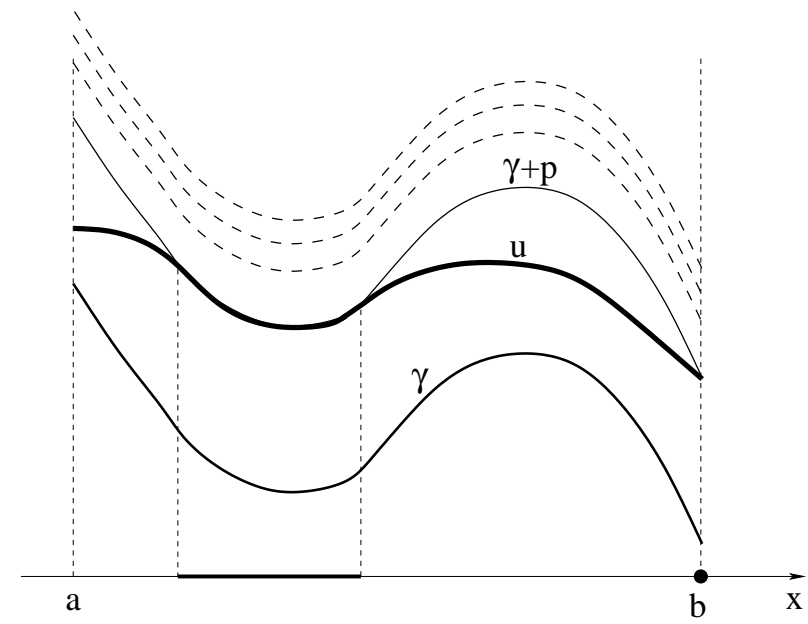

Fig. 1. An example of a solution to the obstacle problem (3.3)-(3.4). Note that $u_{x}(a)=0$, but at the point $b$ one has $u(b)=\gamma(b)+p$ and $\gamma_{x}(b)<u_{x}(b)<0$. If the measure $\mu$ concentrates a positive mass on the boundary $\partial \Omega$, solutions to the variational inequality (3.6) need not satisfy the boundary condition (1.2) in the classical sense. Theorem 3.3 is proved by solving the obstacle problem (3.6) with various choices of $p \geq 0$, and finding a value of $p$ for which (3.1)-(3.2) hold.

Following [24], the problem (3.3)-(3.5) can be reformulated as a differential inequality. Consider the closed convex domain

$$
\mathcal{K}_{p} \doteq\left\{v \in H^{1}(\Omega), \quad v(x) \leq \gamma(x)+p \quad \text { for a.e. } x \in \Omega\right\} .
$$

We then seek $u \in \mathcal{K}_{p}$ such that

$$
\int_{\Omega}[-\nabla u \cdot \nabla(u-v)+f(x, u)(u-v)] d x \geq 0
$$

for all $v \in \mathcal{K}_{p}$. Notice that, in the smooth case,

$$
\begin{aligned}
\int_{\Omega}[-\nabla u & \cdot \nabla(u-v)+f(x, u)(u-v)] d x \\
= & \int_{\Omega}(\Delta u+f(x, u))(u-v) d x-\int_{\partial \Omega}(\mathbf{n} \cdot \nabla u)(u-v) d \sigma .
\end{aligned}
$$

On regions where $u(x)<\gamma(x)+p$, one can choose $v \in \mathcal{K}_{p}$ so that $u-v$ takes both positive and negative signs, thus obtaining (3.5). On the other hand, we can always choose $v \in \mathcal{K}_{p}$ so that $u-v$ is an arbitrary negative function. In this way one obtains (3.4).

2. By the assumptions (A1)-(A2), we can find $0<\delta_{0} \leq \min _{x \in \bar{\Omega}} \gamma(x)$ such that $f(x, u)>0$ for all $x \in \bar{\Omega}$ and $0<u \leq \delta_{0}$. The functions

$$
u^{\text {upper }}(x)=\min \{M, \gamma(x)+p\}, \quad u^{\text {lower }}(x)=\delta_{0}
$$

are thus an upper and a lower solution, respectively. By a comparison argument, the variational inequality admits a solution $u: \bar{\Omega} \mapsto\left[\delta_{0}, M\right]$. By the regularity theory for solutions to variational inequalities with smooth obstacles (see $[9,19,24]$ or Theorem 6.1 in $[26])$, it follows that $u \in W_{l o c}^{2, \infty}(\Omega)$. In particular, $u$ is continuously 
differentiable on the open set $\Omega$. Furthermore, $u$ is globally Lipschitz on the closure $\bar{\Omega}$.

To prove uniqueness, let $u_{1}, u_{2}: \bar{\Omega} \mapsto\left[\delta_{0}, M\right]$ be two solutions. To fix the ideas, assume $u_{1}(y)<u_{2}(y)$ for some $y \in \bar{\Omega}$. By continuity and compactness, there exists $\bar{x} \in \bar{\Omega}$, where the ratio $u_{1} / u_{2}$ attains its minimum, say

$$
0<\lambda \doteq \min _{x \in \bar{\Omega}} \frac{u_{1}(x)}{u_{2}(x)}=\frac{u_{1}(\bar{x})}{u_{2}(\bar{x})}<1 .
$$

Therefore,

$$
0=u_{1}(\bar{x})-\lambda u_{2}(\bar{x}) \leq u_{1}(x)-\lambda u_{2}(x) \quad \text { for all } \quad x \in \bar{\Omega} .
$$

Two cases will be considered.

Case 1. $\bar{x} \in \Omega$ is an interior point. The strict concavity of $f$ implies

$$
f\left(\bar{x}, u_{1}(\bar{x})\right)-\frac{u_{1}(\bar{x})}{u_{2}(\bar{x})} f\left(\bar{x}, u_{2}(\bar{x})\right)>0 .
$$

By continuity, there exist $r, \delta_{0}>0$ small enough so that

$$
-f\left(\bar{x}, u_{1}(x)\right)+\lambda f\left(\bar{x}, u_{2}(x)\right) \leq-\delta_{0} \quad \text { for all } x \in B(\bar{x}, r) .
$$

Consider the function $u_{1}-\lambda u_{2} \in W^{2, \infty}$. For a.e. $x \in B(\bar{x}, r)$ we have

$$
\Delta u_{1}(x)-\lambda \Delta u_{2}(x) \leq-f\left(x, u_{1}(x)\right)-\lambda f\left(x, u_{2}(x)\right) \leq-\delta_{0}<0 .
$$

On the other hand, for points on the boundary of the ball $x \in \partial B(\bar{x}, r)$ one has

$$
u_{1}(x)-\lambda u_{2}(x) \geq 0 .
$$

Together, (3.10) and (3.11) imply the strict inequality

$$
u_{1}(x)-\lambda u_{2}(x)>0 \quad \text { for } \quad|x-\bar{x}|<r .
$$

This yields a contradiction with the first identity in (3.7).

Case 2. $\bar{x} \in \partial \Omega$ is a boundary point. Recalling (3.9), and observing that $u_{1}$ does not touch the obstacle in a neighborhood of $\bar{x}$, we can find $r, \delta_{0}>0$ such that

$$
\begin{gathered}
\mathbf{n}(x) \cdot \nabla\left(u_{1}(x)-\lambda u_{2}(x)\right)=-\lambda \mathbf{n}(x) \cdot u_{2}(x) \geq 0 \quad \text { for all } x \in \partial \Omega \cap B(\bar{x}, r), \\
\Delta u_{1}(x)-\lambda \Delta u_{2}(x) \leq-\delta_{0}<0 \quad \text { for a.e. } x \in \Omega \cap B(\bar{x}, r) .
\end{gathered}
$$

By (3.7) we have

$$
u_{1}(x)-\lambda u_{2}(x) \geq 0 \quad \text { for all } x \in \partial(\Omega \cap B(\bar{x}, r)) .
$$

Hence (3.12)-(3.13) imply

$$
u_{1}(x)-\lambda u_{1}(x)>0 \quad \text { for all } x \in \bar{\Omega}, \quad|x-\bar{x}|<r .
$$

This yields again a contradiction with the first identity in (3.7), thus proving uniqueness. 
3. For each $p \geq 0$, denote by $u^{(p)}$ the unique, strictly positive solution to this obstacle problem. Continuity in $\mathcal{C}^{0}$ can be proved by a comparison argument. Indeed, let $0 \leq \bar{p}<p$. Then $u^{(\bar{p})}$ is a lower solution to the obstacle problem satisfied by $u^{(p)}$. This immediately implies $u^{(\bar{p})}(x) \leq u^{(p)}(x)$. On the other hand, consider a point $\bar{x} \in \bar{\Omega}$, where $\gamma$ attains its (strictly positive) minimum. Define

$$
\lambda \doteq \frac{\gamma(\bar{x})+\bar{p}}{\gamma(\bar{x})+p}, \quad v(x) \doteq \lambda u^{(p)}(x) .
$$

We claim that $v \leq u^{(\bar{p})}$. Indeed, by construction we have

$$
v(x)=\lambda u^{(\bar{p})}(x) \leq \frac{\gamma(\bar{x})+\bar{p}}{\gamma(\bar{x})+p} \cdot(\gamma(x)+p) \leq \gamma(x)+\bar{p}
$$

for all $x \in \bar{\Omega}$. Moreover,

$$
\mathbf{n}(x) \cdot \nabla v(x)=\lambda \mathbf{n}(x) \cdot \nabla v(x) \leq 0 \quad \text { for all } x \in \partial \Omega .
$$

Next, at any point $x \in \Omega$, by the concavity of $f$ w.r.t. $u$ we have

$\lambda(\Delta v+f(x, v(x))) \geq \Delta u^{(p)}(x)+\lambda f\left(x, \lambda u^{(p)}(x)\right) \geq \Delta u^{(p)}(x)+f\left(x, u^{(p)}(x)\right) \geq 0$.

This proves that $v$ is a subsolution, hence $v \leq u^{(\bar{p})}$. The above arguments show that the map $p \mapsto u^{(p)}$ is continuous with values in $\mathcal{C}^{0}(\bar{\Omega})$.

4. Given the solution $u^{(p)}$, define the corresponding measure $\mu^{(p)}$ by setting

$$
\begin{aligned}
\mu^{(p)}(A) & =\int_{A \cap \Omega} \frac{1}{u^{(p)}}\left(\Delta u^{(p)}+f\left(x, u^{(p)}\right)\right) d x-\int_{A \cap \partial \Omega} \frac{1}{u^{(p)}} \mathbf{n}(x) \cdot \nabla u^{(p)} d \sigma \\
& =\int_{A \cap \Omega \cap\{u(x)=\gamma(x)\}} \frac{1}{u^{(p)}}\left(\Delta \gamma+f\left(x, u^{(p)}\right)\right) d x-\int_{A \cap \partial \Omega} \frac{1}{u^{(p)}} \mathbf{n}(x) \cdot \nabla u^{(p)} d \sigma
\end{aligned}
$$

for every Borel set $A$. Notice that the above equality holds because $u \in W_{l o c}^{2, \infty}(\Omega)$. We observe that $\Delta u^{(p)} \in \mathbf{L}^{\infty}(\Omega)$. Indeed, for a.e. $x \in \Omega$, one has

$$
\Delta u^{(p)}(x)=\left\{\begin{array}{cl}
\Delta \gamma(x) & \text { if } u^{(p)}(x)=\gamma(x)+p \\
-f\left(x, u^{(p)}(x)\right) & \text { if } u^{(p)}(x)<\gamma(x)+p .
\end{array}\right.
$$

We claim that the function

$$
p \mapsto \Phi(p) \doteq \mu^{(p)}(\bar{\Omega})
$$

is continuous. Indeed, we can write

$$
\Phi(p)=\Phi_{1}(p)+\Phi_{2}(p) \doteq \int_{\Omega} \frac{\Delta u^{(p)}(x)+f\left(x, u^{(p)}(x)\right)}{\gamma(x)+p} d x-\int_{\partial \Omega} \frac{\mathbf{n} \cdot \nabla u^{(p)}}{u^{(p)}(x)} d \sigma .
$$

By Theorem B in [3], the map $p \mapsto \mathbf{n} \cdot \nabla u^{(p)}$ is continuous with values in $\mathbf{L}^{1}(\partial \Omega)$. Together with the uniform continuity of the map $p \mapsto u^{(p)}$ with values in $\mathcal{C}^{0}(\Omega)$, proved in the previous step, this yields the continuity of the map $p \mapsto \Phi_{2}(p)$.

To prove the continuity of $\Phi_{1}$, let $\bar{p} \geq 0$ and $\varepsilon>0$ be given. Choose $\delta>0$ so that, calling

$$
\Omega_{\delta} \doteq\{x \in \Omega ; d(x, \partial \Omega)<\delta\}
$$




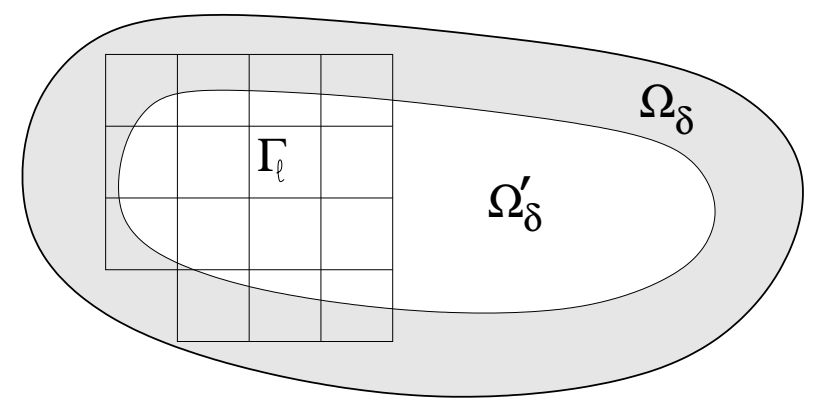

FIG. 2. The decomposition of the domain $\Omega=\Omega_{\delta} \cup \Omega_{\delta}^{\prime}$, where the inner domain is covered by finitely many hypercubes $\Gamma_{\ell}$.

one has

$$
\int_{\Omega_{\delta}}\left|\frac{\Delta \gamma(x)+f(x, \gamma(x)+p)}{\gamma(x)+p}\right| d x<\varepsilon
$$

for all $p$ such that $|p-\bar{p}|<\delta$.

Next, cover the remaining set $\Omega_{\delta}^{\prime} \doteq \Omega \backslash \Omega_{\delta}$ with hypercubes of side $\rho$, choosing $\rho>0$ small enough so that the oscillation of $f, u^{(p)}$ on each such cube $\Gamma_{\ell}$ is $\leq \varepsilon$ (see Figure 2). Namely,

$$
\sup _{x, y \in \Gamma_{\ell}}|f(x, \gamma(x)+p)-f(y, \gamma(y)+p)|<\varepsilon, \quad \sup _{x, y \in \Gamma_{\ell}}\left|u^{(p)}(x)-u^{(p)}(y)\right|<\varepsilon,
$$

for every $\Gamma_{\ell}$ and every $p$ such that $|p-\bar{p}|<\delta$. We now compute

$$
\begin{aligned}
A & \doteq\left|\int_{\Omega_{\delta}^{\prime}} \frac{\Delta u^{(p)}(x)+f\left(x, u^{(p)}(x)\right)}{u^{(p)}(x)}-\frac{\Delta u^{(\bar{p})}(x)+f\left(x, u^{(\bar{p})}(x)\right)}{u^{(\bar{p})}(x)} d x\right| \\
& \leq \int_{\Omega_{\delta}^{\prime}}\left|\frac{f\left(x, u^{(p)}(x)\right)}{u^{(p)}(x)}-\frac{f\left(x, u^{(\bar{p})}(x)\right)}{u^{(\bar{p})}(x)}\right| d x+\sum_{\ell}\left|\int_{\Gamma_{\ell}} \frac{\Delta u^{(p)}(x)}{u^{(p)}(x)}-\frac{\Delta u^{(\bar{p})}(x)}{u^{(\bar{p})}(x)} d x\right| \\
& \doteq A_{1}+A_{2} .
\end{aligned}
$$

As $p \rightarrow \bar{p}$, the uniform convergence $u^{(p)} \rightarrow u^{(\bar{p})}$ implies that $A_{1} \rightarrow 0$. To estimate $A_{2}$, call $u_{\ell}^{(p)}$ and $u_{\ell}^{(\bar{p})}$ the average values of $u^{(p)}$ and $u^{(\bar{p})}$ on the cube $\Gamma_{\ell}$, respectively. For each $\ell$ we now have

$$
\begin{aligned}
& \left|\int_{\Gamma_{\ell}} \frac{\Delta u^{(p)}(x)}{u^{(p)}(x)}-\frac{\Delta u^{(\bar{p})}(x)}{u^{(\bar{p})}(x)} d x\right| \\
& \quad \leq\left|\int_{\Gamma_{\ell}} \frac{\Delta u^{(p)}(x)-\Delta u^{(\bar{p})}(x)}{u_{\ell}^{(\bar{p})}} d x\right|+\int_{\Gamma_{\ell}}\left|\Delta u^{(p)}(x)\right| d x \cdot \sup _{x \in \Gamma_{\ell}}\left|\frac{1}{u^{(p)}(x)}-\frac{1}{u_{\ell}^{(\bar{p})}}\right| \\
& \quad \leq\left(\min _{x \in \bar{\Omega}} u^{(\bar{p})}\right)^{-1} \cdot\left|\int_{\partial \Gamma_{\ell}} \frac{\nabla u^{(p)}(x)-\nabla u^{(\bar{p})}(x)}{u_{\ell}^{(\bar{p})}} d x\right| \\
& \quad+\int_{\Gamma_{\ell}}\left|\Delta u^{(p)}(x)\right| d x \cdot \sup _{x \in \Gamma_{\ell}}\left|\frac{1}{u^{(p)}(x)}-\frac{1}{u_{\ell}^{(\bar{p})}}\right| .
\end{aligned}
$$

We now observe that the quantities

$$
\left(\min _{x \in \bar{\Omega}} u^{(\bar{p})}\right)^{-1}, \quad \int_{\Omega}\left|\Delta u^{(p)}(x)\right| d x
$$

Copyright (c) by SIAM. Unauthorized reproduction of this article is prohibited. 
remain uniformly bounded. Indeed, the first bound is a consequence of the strict inequality $f_{u}(x, 0)>0$ in (1.3) and of (1.8). By (3.5), the second bound follows from

$$
\int_{\Omega}\left|\Delta u^{(p)}(x)\right| d x \leq \int_{\Omega} \max \left\{|\Delta \gamma(x)|,\left|f\left(x, u^{(p)}(x)\right)\right|\right\} d x .
$$

Since $A_{2}$ is a sum over finitely many cubes, we conclude that

$$
A_{2}=\mathcal{O}(1) \cdot \sum_{\ell}\left|\int_{\partial \Gamma_{\ell}} \frac{\nabla u^{(p)}(x)-\nabla u^{(\bar{p})}(x)}{u_{\ell}^{(\bar{p})}} d x\right|+\mathcal{O}(1) \cdot \sup _{x \in \Gamma_{\ell}}\left|\frac{1}{u^{(p)}(x)}-\frac{1}{u_{\ell}^{(\bar{p})}}\right| .
$$

Given $\varepsilon>0$, we first choose the diameter of each cube small enough, so that

$$
\sup _{\ell} \sup _{x \in \Gamma_{\ell}}\left|\frac{1}{u^{(p)}(x)}-\frac{1}{u_{\ell}^{(\bar{p})}}\right|<\varepsilon
$$

By a result in [3], the map $p \mapsto \mathbf{n} \cdot \nabla u^{(p)}$ is continuous with values in $\mathbf{L}^{1}(\partial \Omega)$. Letting $p \rightarrow \bar{p}$, each term of the summation on the right-hand side thus converges to zero. We conclude that, as $p \rightarrow \bar{p}$, both terms $A_{1}, A_{2}$ approach zero, proving the continuity of the map $p \mapsto \Phi(p)$.

5. Using the fact that $\Phi$ is continuous, we can now conclude as follows.

For $p>0$ large enough, we have $\gamma(x)+p>M$ for all $x \in \bar{\Omega}$. Hence the measure $\mu^{(p)}$ vanishes and $\Phi(p)=0$. Two cases can now arise.

Case 1. $\Phi(0) \geq \kappa$. In this case, by continuity there exists some value $p \geq 0$ such that $\Phi(p)=\kappa$. In this case, the measure $\mu^{(p)}$ defined at (3.14) satisfies all requirements.

Case 2. $\Phi(0)<\kappa$. In this case, we simply take $p=0$. The measure $\mu^{(p)}$ defined at (3.14) again satisfies our requirements.

Remark 3.4. The uniqueness of the equilibrium solution is left as an open question. According to the above analysis, uniqueness would follow from the strict monotonicity of the map $p \mapsto \Phi(p)$ in (3.16). However, there is no obvious reason for $\Phi$ to be strictly decreasing. In this context, it is worth observing that the coincidence sets

$$
\Sigma^{(p)} \doteq\left\{x \in \bar{\Omega} ; \quad u^{(p)}(x)=\gamma(x)+p\right\}
$$

may not become smaller as $p$ increases.

Remark 3.5. For any given $\kappa>0$, the total payoff achieved by competing players cannot be greater than the maximum payoff achieved by one single player with total harvesting capacity $\kappa$. From the above analysis it follows that, whenever

$$
\kappa>\max _{p \in[0, M]} \mu^{(p)}(\bar{\Omega}),
$$

the fish density must decrease to a level where the profit for every competing agent is exactly zero. Namely,

$$
u(x) \leq \gamma(x), \quad x \in \bar{\Omega}
$$

This is a form of the so-called "tragedy of commons": because of competition, the natural resources are depleted to a level where no profit is possible [21].

If harvesting is cheap, i.e., if the harvesting cost $\gamma(x)>0$ is small, then the total amount of fish caught will be small as well. Indeed, the concavity assumption in (1.3) implies

$$
\int_{\bar{\Omega}} u d \mu=\int_{\Omega} f(x, u(x)) d x \leq \int_{\Omega} f_{u}(x, 0) \gamma(x) d x .
$$

Copyright $@$ by SIAM. Unauthorized reproduction of this article is prohibited. 
4. Players with different costs. In the previous sections we assumed that all fishermen had the same cost $\gamma(x)$ for fishing at the location $x$. This may not be a realistic assumption. For example, think of $N$ groups of fishermen, located at coastal towns $y_{1}, y_{2}, \ldots, y_{N} \in \bar{\Omega}$. To members of the $i$ th group, the cost $\gamma_{i}(x)$ for fishing at a point $x$ will depend on the distance from $x$ to the harbor, say

$$
\gamma_{i}(x)=\sqrt{1+\left|x-y_{i}\right|^{2}}, \quad i=1, \ldots, N .
$$

We shall extend Definition 3.1 to cover this more general situation. In the following, for $i=1, \ldots, N$, we call $\kappa_{i}$ the joint harvesting capability of all fishermen in the $i$-th group, while $\mu_{i}$ will be the combined harvesting effort of all members of the $i$-th group.

Definition 4.1 (Nash equilibrium with different groups of small players). Consider a harvesting game governed by (1.1)-(1.2), with $N$ groups of players. Let $\kappa_{i}>0$ be the harvesting capability of the ith group, and let $\gamma_{i}(x)$ be the corresponding cost for fishing at $x$.

We say that $\left(u, \mu_{1}, \ldots, \mu_{N}\right)$ is an equilibrium solution to the harvesting game provided that:

- Each $\mu_{i}$ is a positive measure on $\bar{\Omega}$, with $\mu_{i}(\bar{\Omega}) \leq \kappa_{i}$.

- $u$ is a strictly positive solution to the elliptic equation (1.1) with Neumann boundary conditions (1.2), where $\mu=\mu_{1}+\cdots+\mu_{N}$.

- There exist constants $p_{i} \geq 0$ such that

$$
\begin{array}{ll}
u(x)-\gamma_{i}(x)=p_{i} & \text { on } \operatorname{Supp}\left(\mu_{i}\right), \\
u(x)-\gamma_{i}(x) \leq p_{i} & \text { for all } x \in \Omega .
\end{array}
$$

Moreover, one notes the implication

$$
p_{i}>0 \quad \Longrightarrow \quad \mu_{i}(\bar{\Omega})=\kappa_{i} .
$$

THEOREM 4.2. Let the assumptions (A1)-(A2) hold, and assume that all cost functions $\gamma_{i}, i=1, \ldots, N$ satisfy the same assumptions as in (A4). Then the harvesting game with with different groups of small players has an equilibrium solution.

Proof. 1. For every $N$-tuple $p=\left(p_{1}, \ldots, p_{N}\right) \in[0, M]^{N}$, call $u^{(p)}$ the solution to the semilinear problem (3.3)-(3.5), where the constraint $u(x) \leq \gamma(x)+p$ is replaced by

$$
u(x) \leq \gamma^{(p)}(x) \doteq \min _{i}\left(\gamma_{i}(x)+p_{i}\right)
$$

By arguments very similar to those in section 3, one can prove that the this problem has a unique positive solution, which depends continuously on $p$. In more detail, the existence of a solution is proved by solving the variational inequality (3.6) on the domain

$$
\mathcal{K}_{\left(p_{1}, \ldots, p_{N}\right)} \doteq\left\{v \in H^{1}(\Omega), \quad v(x) \leq \min _{i}\left(\gamma_{i}(x)+p_{i}\right) \quad \text { for a.e. } x \in \Omega\right\} .
$$

We now observe that the solution $u$ has the same regularity as in the previous case. Namely, $u$ lies in $W_{l o c}^{2, \infty}(\Omega)$ and is Lipschitz on the closure $\bar{\Omega}$. Indeed, in order to 
apply the regularity theorems in $[24,26]$ one only needs to check that the positive part of the measure $\Delta \gamma^{(p)}+f$ is absolutely continuous w.r.t. Lebesgue measure, with density in $\mathbf{L}^{\infty}$. This is clearly true because the source term $f$ is bounded above and below, and moreover, for any choice of $\left(p_{1}, \ldots, p_{N}\right)$, one has

$$
\left\|\left(\Delta \gamma^{(p)}\right)^{+}\right\|_{\mathbf{L}^{\infty}(\Omega)} \leq \max _{i} \sup _{x \in \Omega}\left(\Delta \gamma_{i}(x)\right)^{+} \leq C .
$$

By the regularity property $\Delta u \in \mathbf{L}^{\infty}(\Omega)$ and the strict convexity of $f$ w.r.t. $u$, the same arguments as in step $\mathbf{2}$ of the proof of Theorem 3.3 yield the uniqueness of the solution $u^{(p)}$ to the obstacle problem.

2. Let $\mu^{(p)}$ be the positive measure defined as in (3.14). In the present setting, $\mu^{(p)}$ describes the combined harvesting effort of all fishermen together. Repeating the arguments in steps $\mathbf{3}$ and $\mathbf{4}$ of the proof of Theorem 3.3, we obtain

(i) the continuity of the map $p=\left(p_{1}, \ldots, p_{N}\right) \mapsto u^{(p)}$, as a map from $\mathbb{R}^{N}$ into $\mathcal{C}^{0}(\bar{\Omega})$ and

(ii) the continuity of the map $p \mapsto \mu^{(p)}(\bar{\Omega})$.

In particular, by (i) it follows that the multifunctions

$$
p \mapsto \Sigma_{i}^{(p)} \doteq\left\{x \in \bar{\Omega} ; u^{(p)}(x)=\gamma_{i}(x)+p_{i}\right\}, \quad i=1, \ldots, N,
$$

have a closed graph.

3. For every $p=\left(p_{1}, \ldots, p_{N}\right)$, in connection with the positive measure $\mu^{(p)}$, define the set of coefficients

$$
\begin{gathered}
\Lambda^{(p)} \doteq\left\{\left(\lambda_{1}, \ldots, \lambda_{N}\right) \text {; there exist positive measures } \mu_{1}, \ldots, \mu_{N} \text { such that } \mu_{i}(\bar{\Omega})=\lambda_{i},\right. \\
\left.\mu^{(p)}=\mu_{1}+\cdots+\mu_{N}, \operatorname{Supp}\left(\mu_{i}\right) \subseteq\left\{x ; u^{(p)}(x)=\gamma_{i}(x)+p_{i}\right\}, \quad i=1, \ldots, N\right\} .
\end{gathered}
$$

We claim that the map $p \mapsto \Lambda^{(p)}$ defined at (4.7) is an upper semicontinuous multifunction with nonempty, closed, convex values.

To prove that each set $\Lambda^{(p)}$ is nonempty, consider the coincidence sets $\Sigma_{1}^{(p)}, \ldots, \Sigma_{N}^{(p)}$ introduced at (4.6). Define

$$
\lambda_{1} \doteq \mu^{(p)}\left(\Sigma_{1}^{(p)}\right), \quad \lambda_{i} \doteq \mu^{(p)}\left(\Sigma_{i}^{(p)} \backslash \Sigma_{i-1}^{(p)}\right), \quad i=2, \ldots, N .
$$

Then $\left(\lambda_{1}, \ldots, \lambda_{N}\right) \in \Lambda^{(p)}$.

To prove that $\Lambda^{(p)}$ is convex, assume $\left(\lambda_{1}, \ldots, \lambda_{N}\right),\left(\tilde{\lambda}_{1}, \ldots, \tilde{\lambda}_{N}\right) \in \Lambda^{(p)}$. Let

$$
\mu_{1}+\cdots+\mu_{N}=\mu^{(p)}=\tilde{\mu}_{1}+\cdots+\tilde{\mu}_{N}
$$

be the corresponding measures. For any $\sigma \in[0,1]$ and $i \in\{1, \ldots, N\}$, the measure

$$
\mu_{i}^{\sigma}=\sigma \mu_{i}+(1-\sigma) \tilde{\mu}_{i}
$$

is supported on the set where $u(x)=c_{i}(x)+p_{i}$. Moreover,

$$
\mu_{1}^{\sigma}+\cdots+\mu_{N}^{\sigma}=\mu^{(p)} .
$$

This shows that each set $\Lambda^{(p)}$ is convex. 
4. To prove upper semicontinuity, we need to check that the graph of $p \mapsto \Lambda^{(p)}$ is closed. Toward this goal, we recall that the multifunctions $p \mapsto \Sigma_{i}^{(p)}$ introduced at (4.6) have a closed graph.

According to (3.14), the measure $\mu^{(p)}$ is the sum of two parts. The restriction of $\mu^{(p)}$ to the open set $\Omega$ is absolutely continuous with density

$$
\phi(x) \doteq \frac{\Delta u^{(p)}(x)+f\left(x, u^{(p)}(x)\right)}{u^{(p)}(x)}=\frac{\Delta \gamma^{(p)}(x)+f\left(x, u^{(p)}(x)\right)}{u^{(p)}(x)} \cdot \chi_{\left\{u^{(p)}(x)=\gamma^{(p)}(x)\right\}}
$$

w.r.t. Lebesgue measure, while the restriction of $\mu^{(p)}$ to the boundary $\partial \Omega$ has density

$$
\psi(x) \doteq-\frac{\mathbf{n}(x) \cdot \nabla u^{(p)}(x)}{u^{(p)}(x)}
$$

w.r.t. $(d-1)$-dimensional surface measure. In (4.8) we denote by $\chi_{A}$ the characteristic function of a set $A$, while $\gamma^{(p)}$ is the obstacle function introduced at (4.4).

We now consider a convergent sequence

$$
\left(p_{1, n}, \ldots, p_{N, n}\right) \rightarrow\left(p_{1}, \ldots, p_{N}\right) .
$$

Let $\left(\lambda_{1, n}, \ldots \lambda_{N, n}\right) \in \Lambda^{\left(p_{n}\right)}$ for all $n \geq 1$, and assume the convergence $\left(\lambda_{1, n}, \ldots \lambda_{N, n}\right) \rightarrow$ $\left(\lambda_{1}, \ldots \lambda_{N}\right)$. We need to show that $\left(\lambda_{1}, \ldots \lambda_{N}\right) \in \Lambda^{(p)}$.

By the uniform convergence

$$
\gamma^{\left(p_{n}\right)}(x) \doteq \min _{i}\left(\gamma_{i}(x)+p_{i, n}\right) \rightarrow \min _{i}\left(\gamma_{i}(x)+p_{i}\right) \doteq \gamma^{(p)}(x), \quad u^{\left(p_{n}\right)} \rightarrow u^{(p)},
$$

the convergence of the corresponding densities in (4.8)-(4.9) follows, namely

$$
\phi_{n} \rightarrow \phi, \quad \psi_{n} \rightarrow \psi,
$$

in $\mathbf{L}^{1}(\Omega)$ and in $\mathbf{L}^{1}(\partial \Omega)$, respectively. Here $\phi, \psi$ are the densities of the limit measure $\mu^{(p)}$.

For each $n \geq 1$, by assumption there exist positive measures $\mu_{1, n}, \ldots, \mu_{N, n}$ such that

$\mu_{i, n}(\bar{\Omega})=\lambda_{i, n}, \quad \operatorname{Supp}\left(\mu_{i, n}\right) \subseteq\left\{x ; u^{\left(p_{n}\right)}(x)=\gamma_{i}(x)+p_{i}\right\}, \quad \mu^{\left(p_{n}\right)}=\mu_{1, n}+\cdots+\mu_{N, n}$.

By possibly taking a subsequence, one has the weak convergence $\left(\mu_{1, n}, \ldots, \mu_{N, n}\right) \rightarrow$ $\left(\mu_{1}, \ldots, \mu_{N}\right)$. The conditions (4.10), together with the upper semicontinuity of the sets $\Sigma_{i}$ in (4.6), imply

$\mu_{i}(\bar{\Omega})=\lambda_{i}, \quad \operatorname{Supp}\left(\mu_{i}\right) \subseteq\left\{x ; u^{(p)}(x)=\gamma_{i}(x)+p_{i}\right\}, \quad \mu^{(p)}=\mu_{1}+\cdots+\mu_{N}$, proving that $\left(\lambda_{1}, \ldots, \lambda_{N}\right) \in \Lambda^{(p)}$, as claimed. field

5. On the $N$-dimensional cube $\Gamma \doteq[0, M]^{N}$ we now define the multivalued vector

$$
\mathbf{v}(p)=\left\{\left(\lambda_{1}, \ldots, \lambda_{N}\right)-\left(\kappa_{1}, \ldots, \kappa_{N}\right) ; \quad\left(\lambda_{1}, \ldots, \lambda_{N}\right) \in \Lambda^{(p)}\right\} .
$$

By the previous step, this is an upper semicontinuous map with compact, convex values. By the general theory of variational inequalities [20,24], there exists a point $p^{*} \in \Gamma$ and a vector

$$
\mathbf{v}^{*}=\left(v_{1}^{*}, \ldots, v_{N}^{*}\right)=\left(\lambda_{1}^{*}-\kappa_{1}, \ldots, \lambda_{N}^{*}-\kappa_{N}\right) \in \mathbf{v}\left(p^{*}\right)
$$


such that

$$
\left\langle\mathbf{v}^{*}, p-p^{*}\right\rangle \leq 0 \quad \text { for all } p \in \Gamma .
$$

We claim that this provides the required solution. Indeed, consider any $i \in\{1, \ldots, N\}$. If $\lambda_{i}^{*}=\kappa_{i}$ we are done. Otherwise we consider two cases.

Case 1. $\lambda_{i}^{*}<\kappa_{i}$. We then use (4.11), choosing the vector $p=\left(p_{1}^{*}, \ldots, p_{i-1}^{*}, 0, p_{i+1}^{*}\right.$, $\left.\ldots, p_{N}^{*}\right)$. This yields

$$
\left\langle\mathbf{v}^{*}, p-p^{*}\right\rangle=-v_{i}^{*} p_{i}^{*}=-\left(\lambda_{i}^{*}-\kappa_{i}\right) p_{i}^{*} \leq 0 .
$$

This implies $p_{i}^{*}=0$, because otherwise a contradiction is obtained.

Case 2. $\lambda_{i}^{*}>\kappa_{i}$. We then use (4.11), choosing the vector $p=\left(p_{1}^{*}, \ldots, p_{i-1}^{*}\right.$, $\left.M, p_{i+1}^{*}, \ldots, p_{N}^{*}\right)$. This yields

$$
\left\langle\mathbf{v}^{*}, p-p^{*}\right\rangle=-v_{i}^{*}\left(M-p_{i}^{*}\right)=\left(\lambda_{i}^{*}-\kappa_{i}\right)\left(M-p_{i}^{*}\right) \leq 0 .
$$

This implies $p_{i}^{*}=M$, because otherwise a contradiction is obtained.

On the other hand, if $p_{i}^{*}=M$, then the measure $\mu_{i}$ has total mass

$$
\mu_{i}(\bar{\Omega})=\lambda_{i}^{*}>\kappa_{i}>0
$$

and is supported in the set

$$
\left\{x \in \bar{\Omega} ; \quad u(x) \geq \gamma_{i}(x)+M\right\} .
$$

Since $u(x) \leq M$ for all $x$, the above set is empty, reaching a contradiction with (4.12).

The previous arguments show that, for every $i=1, \ldots, N$, either $p_{i}^{*}=0$ or else $\mu_{i}(\bar{\Omega})=\kappa_{i}$, as required by (4.3). This completes the proof.

5. A continuum of different players. We consider here a continuum of fishermen, labeled by a variable $\theta \in[0, \kappa]$. We let $\gamma(x, \theta)$ be the cost to fisherman $\theta$ for harvesting at $x$. Throughout, we assume the following:

(A5) The map $\theta \mapsto \gamma(\cdot, \theta)$ is measurable and uniformly bounded, from $[0, \kappa]$ into $\mathcal{C}^{2}(\bar{\Omega})$. Moreover,

$$
\gamma(x, \theta) \geq c_{0}>0
$$

for all $x \in \bar{\Omega}$ and $\theta \in[0, \kappa]$.

By a Lusin type theorem, this implies that, for every $\varepsilon>0$, there exists a closed set $K_{\varepsilon} \subset[0, \kappa]$, with meas $\left(K_{\varepsilon}\right)>\kappa-\varepsilon$, such that the restriction $\gamma: K_{\varepsilon} \mapsto \mathcal{C}^{2}(\bar{\Omega})$ is continuous.

DEFINITION 5.1 (Nash equilibrium with infinitely many, different small players). We say that $\left(u, \mu^{\theta}\right)$ is a generalized equilibrium solution to the harvesting game (1.1)-(1.2) for a continuum of players with costs $\gamma=\gamma(x, \theta)$ provided that:

(i) $u$ is a solution to the elliptic equation (1.1) with Neumann boundary conditions (1.2), where $\mu$ is the measure defined by

$$
\mu(V) \doteq \int_{0}^{\kappa} \mu^{\theta}(V) d \theta
$$

Copyright $@$ by SIAM. Unauthorized reproduction of this article is prohibited. 
(ii) For a.e. $\theta \in[0, \kappa], \mu^{\theta}$ is a positive measure on $\bar{\Omega}$ such that

$$
\begin{aligned}
& \operatorname{Supp}\left(\mu^{\theta}\right) \subseteq\left\{x \in \bar{\Omega} ; u(x)-\gamma(x, \theta)=\max _{y \in \bar{\Omega}}\{u(y)-\gamma(y, \theta)\} \geq 0\right\}, \\
& 5.5) \quad \max _{y \in \bar{\Omega}}\{u(y)-\gamma(y, \theta)\}>0 \quad \Longrightarrow \quad \mu^{\theta}(\bar{\Omega})=1 .
\end{aligned}
$$

Remark 5.2. As in the previous sections, here $\kappa$ corresponds to the maximum harvesting capacity. For all fishermen, the payoff from harvesting at a point $x$ is proportional to the density of fish at $x$, namely $u(x)$. On the other hand, the cost to player $\theta$ for harvesting at $x$ is $\gamma(x, \theta)$.

According to (5.3)-(5.5), any fisherman $\theta \in[0, \kappa]$ can choose to work during a fraction $\mu^{\theta}(\bar{\Omega})$ of his time, and remain idle for the remaining portion $1-\mu^{\theta}(\bar{\Omega})$. If $\mu^{\theta}(\bar{\Omega})>0$, he will harvest at points $x \in \bar{\Omega}$, distributed according to a measure $\mu^{\theta}$, supported within the set where the profit $u(x)-\gamma(x, \theta)$ attains a nonnegative maximum.

By (5.5), if there are points $x \in \bar{\Omega}$ where this profit is strictly positive, then fisherman $\theta$ will work full time, i.e., $\mu^{\theta}(\bar{\Omega})=1$.

THEOREM 5.3. Let the assumptions (A1)-(A2) hold, together with (A5). Then the harvesting game with infinitely many small players has a generalized equilibrium solution.

Proof. We shall use Theorem 4.2 together with an approximation argument.

1. Let $\varepsilon>0$ be given. By the assumption (A5) there exists a closed set $K_{\varepsilon} \subset[0, \kappa]$ with meas $\left(K_{\varepsilon}\right)>\kappa-\varepsilon$ such that the map

$$
\theta \mapsto \gamma(\cdot, \theta)
$$

is continuously restricted to $K_{\varepsilon}$. Hence it can be approximated with a function which is piecewise constant w.r.t. $\theta$.

More precisely, there exist cost functions $\gamma_{1}, \ldots, \gamma_{N}$ and numbers

$$
0 \doteq \theta_{0}<\theta_{1}<\ldots, \theta_{N}=\kappa
$$

such that

$$
\left.\left.\left|\gamma(x, \theta)-\gamma_{i}(x)\right|<\varepsilon \quad \text { for all } \theta \in\right] \theta_{i-1}, \theta_{i}\right] \cap K_{\varepsilon} .
$$

By Theorem 4.2, the harvesting game with $N$ groups of fishermen having costs $\gamma_{i}$ and sizes

$$
\kappa_{i} \doteq \operatorname{meas}\left(\left[\theta_{i-1}, \theta_{i}\right] \cap K_{\varepsilon}\right)
$$

has at least one Nash equilibrium solution. Let $\left(\mu_{1}, \ldots, \mu_{N}\right)$ be the measures on $\bar{\Omega}$ corresponding to this solution. Each of these measures can be represented as the push-forward of Lebesgue measure on $\left.\left.\Theta_{i} \subseteq\right] \theta_{i-1}, \theta_{i}\right] \cap K_{\varepsilon}$ through some measurable $\operatorname{map} \xi_{i}(\cdot)$.

Call $\Theta \doteq \cup_{i} \Theta_{i}$, and let $\xi: \Theta \mapsto \bar{\Omega}$ be the map whose restriction to $\Theta_{i}$ coincides with $\xi_{i}$. We then define the family of measures $\left\{\mu^{\theta} ; \theta \in[0, \kappa]\right\}$ by setting the following: 
- If $\theta \in \Theta$, then $\mu^{\theta}$ is a unit mass at the point $\xi(\theta)$.

- If $\theta \notin \Theta$, then $\mu^{\theta}$ is the zero measure.

2. We now take a decreasing sequence $\varepsilon_{n} \downarrow 0$. For each $n$, following the above procedure we construct an approximate equilibrium solution. Let $u_{n}(\cdot)$ be the corresponding sequence of fish densities. The uniform bound $\|\gamma(\cdot, \theta)\|_{\mathcal{C}^{2}} \leq C$ for all $\theta \in[0, \kappa]$ implies that all $u_{n}$ have uniformly bounded $\mathcal{C}^{1}$ norm. In particular, they are uniformly Lipschitz continuous on $\bar{\Omega}$. By possibly taking a subsequence we can assume the uniform convergence $u_{n} \rightarrow u$. Moreover, without loss of generality we can assume that the domains $K_{\varepsilon_{n}} \subset[0, \kappa]$ are monotone increasing:

$$
K_{\varepsilon_{n}} \subseteq K_{\varepsilon_{n+1}}
$$

By construction, a.e. $\theta \in[0, \kappa]$ is a Lebesgue point of some $K_{\varepsilon_{n}}$.

For each $n \geq 1$, consider the measure $\mu_{n}$ on the product space $[0, \kappa] \times \bar{\Omega}$ defined by

$$
\mu_{n}(A) \doteq \int_{0}^{\kappa} \mu_{n}^{\theta}(\{x \in \bar{\Omega} ; \quad(x, \theta) \in A\}) d \theta .
$$

By extracting a further subsequence, we can assume the weak convergence $\mu_{n} \rightarrow \mu_{\infty}$. By a disintegration theorem (see section 2.5 in [1]), this limit measure $\mu_{\infty}$ on $[0, \kappa] \times \bar{\Omega}$ can be represented in the form

$$
\mu_{\infty}(A) \doteq \int_{0}^{\kappa} \mu^{\theta}(\{x \in \bar{\Omega} ; \quad(x, \theta) \in A\}) d \theta .
$$

In the remaining part of the proof we show that, inserting the above measures $\mu^{\theta}$ into (5.2)-(5.5), all requirements of Definition 5.1 are satisfied.

3. We observe that, since

$$
\mu_{n}(V \times \bar{\Omega}) \leq \operatorname{meas}(V)
$$

for every Borel subset $V \subseteq[0, \kappa]$, this implies that the same holds for the limit measure $\mu_{\infty}$. Hence

$$
\mu^{\theta}(\bar{\Omega}) \leq 1
$$

for all $\theta \in[0, \kappa]$. This proves $(5.3)$.

4. Next, we claim that $u$ provides the solution to (1.1)-(1.2), in connection with the measure $\mu$ defined at (5.2). This is an immediate consequence of the weak convergence $\mu_{n} \rightarrow \mu$ and the uniform convergence $u_{n} \rightarrow u$.

To prove (5.4) and (5.5), consider a value $\theta^{*} \in[0, \kappa]$ which is a density point of some set $K_{\varepsilon_{m}}$.

Then the map $\theta \mapsto \gamma(\cdot, \theta)$ is quasi-continuous at $\theta^{*}$, namely

$$
\lim _{r \rightarrow 0} \frac{1}{2 r} \int_{\theta^{*}-r}^{\theta^{*}+r}\left\|\gamma(\cdot, \theta)-\gamma\left(\cdot, \theta^{*}\right)\right\|_{\mathcal{C}^{2}(\Omega)}=0 .
$$

By continuity, for any given $\varepsilon>0$ and all $n \geq 1$ large enough we have

$$
\operatorname{Supp}\left(\mu_{n}^{\theta}\right) \subseteq\left\{x \in \bar{\Omega} ; u_{n}(x)-\gamma(x, \theta)-\varepsilon \leq \max _{y \in \bar{\Omega}}\left[u_{n}(y)-\gamma(y, \theta)\right]\right\}
$$


provided $\theta \in K_{\varepsilon_{n}}$.

By the continuity of $\gamma$ on $K_{\varepsilon_{n}}$, and the uniform convergence $u_{n} \rightarrow u$, this yields

$$
\operatorname{Supp}\left(\mu^{\theta}\right) \subseteq\left\{x \in \bar{\Omega} ; \quad u_{n}(x)-\gamma(x, \theta)-\varepsilon \leq \max _{y \in \bar{\Omega}}[u(y)-\gamma(y, \theta)]\right\}
$$

for all $\theta$ which are density points of some set $K_{\varepsilon_{m}}$. Hence this is true for a.e. $\theta \in[0, \kappa]$. Since $\varepsilon>0$ can be chosen arbitrarily small, the conclusion holds for a.e. $\theta$. This proves (5.4).

6. Finally, let $\theta^{*}$ be a density point of some set $K_{\varepsilon_{m}}$, and assume that

$$
\max _{y \in \bar{\Omega}}\left[u(y)-\gamma\left(y, \theta^{*}\right)\right]>0 .
$$

Then

$$
\max _{y \in \bar{\Omega}}\left(u_{n}(y)-\gamma\left(y, \theta^{*}\right)\right)>0
$$

for all $n$ sufficiently large. In turn, this implies

$$
\mu_{n}^{\theta}(\bar{\Omega})=1
$$

for all $n$ large and all $\theta \in K_{\varepsilon_{m}}$ sufficiently close to $\theta^{*}$. Taking a weak limit, this yields $\mu^{\theta^{*}}(\bar{\Omega})=1$, proving $(5.5)$.

Remark 5.4. By a theorem of Skorokhod (see, for example, [4, pp. 185-187]), the measure $\mu$ constructed in Theorem 3.3 can be represented as the push forward of the Lebesgue measure on an interval $\left[0, \kappa^{\prime}\right]$, with $\kappa^{\prime} \leq \kappa$. In other words, there exists a measurable map $\phi:\left[0, \kappa^{\prime}\right] \mapsto \bar{\Omega}$ such that

$$
\mu(V)=\operatorname{meas}\left(\left\{\theta \in\left[0, \kappa^{\prime}\right] ; \phi(\theta) \in V\right\}\right)
$$

for every Borel set $V \subset \mathbb{R}^{d}$. We can think of $\theta$ as a Lagrangian variable labeling the various fishermen. In the equilibrium solution, each fisherman $\theta \in\left[0, \kappa^{\prime}\right]$ works full time at the single location $\phi(x)$, while if $\kappa^{\prime}<\kappa$, all fishermen $\left.\left.\theta \in\right] \kappa^{\prime}, \kappa\right]$ remain idle.

In contrast with the results proved in the previous sections, Theorem 5.3 only establishes the existence of an equilibrium solution within generalized strategies. To each player $\theta \in[0, \kappa]$ we associate a positive measure $\mu^{\eta}$ with total mass $\leq 1$. In general, it is not possible to choose $\mu^{\eta}$ a Dirac mass (meaning the harvesting effort is concentrated at a single point) or the zero measure (meaning that the fisherman remains idle). As shown by the following example, this is not a shortcoming of the analysis, but reflects the nature of the problem.

Example 5.5. On the unit circle $\Omega=\left\{x \in \mathbb{R}^{2} ;|x|<1\right\}$, consider a concave function $f=f(u)$ such that

$$
f(0)=f(3)=0, \quad\left\{\begin{array}{cc}
f(u)=1 / 2 & \text { if } \quad u \in[1,2], \\
f^{\prime \prime}(u) \leq 0 & \text { if } \quad u \in[0,3] .
\end{array}\right.
$$

As cost functions, working in polar coordinates $(r, \alpha)$, assume

$$
\gamma(r, \alpha ; \theta) \doteq 2-r^{2} \cos ^{2}(\alpha-\theta), \quad \theta \in[0, \pi] .
$$


Then the function

$$
u(r, \alpha)=1+\frac{1-r^{2}}{8}
$$

satisfies

$$
\left\{\begin{aligned}
\Delta u+f(u) & =0, & & x \in \Omega, \\
\mathbf{n} \cdot \nabla u & =-1 / 4, & & x \in \partial \Omega .
\end{aligned}\right.
$$

The equilibrium solution is provided by the family of measures $\left\{\mu^{\theta} ; \theta \in[0, \pi]\right\}$, where $\mu^{\theta}$ is the atomic measure containing two masses, each of size $1 / 4$, namely the two points

$$
P^{\theta}=(\cos \theta, \sin \theta), \quad Q^{\theta}=(-\cos \theta,-\sin \theta) .
$$

Notice that none of these measures $\mu^{\theta}$ is a probability measure. We observe that, for each $\theta \in[0, \pi]$, the maximum of the profit $u(\cdot)-\gamma(\cdot, \theta)$ to player $\theta$ is

$$
\max _{r \leq 1, \alpha \in[0,2 \pi]}\left\{1+\frac{1-r^{2}}{8}-\left(2-r^{2} \cos ^{2}(\alpha-\theta)\right)\right\}=0
$$

and it is attained precisely at the two points $(r, \alpha)=(1, \theta)$ or $(1, \theta+\pi)$.

The corresponding measure $\mu$ in (5.2) is uniformly distributed along the unit circumference, with density $1 / 4$ w.r.t. arc length. This is indeed consistent with (5.7).

We remark that there is no way to obtain the measure $\mu$ as the push-forward of the Lebesgue measure on a subset $\Theta \subseteq[0, \pi]$ by some (single-valued) measurable map $\theta \mapsto \xi(\theta)$ such that

$$
\xi(\theta) \in\left\{P^{\theta}, Q^{\theta}\right\}
$$

for every $\theta \in \Theta$. Indeed, as $\theta$ varies in $] 0, \pi]$, the sets $\left\{P^{\theta}, Q^{\theta}\right\}$ are all mutually disjoint. Hence, for any map $\xi:[0, \pi] \mapsto \partial \Omega$, the corresponding measure $\mu^{\xi}$ is given by

$$
\mu^{\xi}(V)=m_{1}\left(V \cap A_{\xi}\right),
$$

where $m_{1}$ is the 1-dimensional measure on $\partial \Omega$, and $A_{\xi}=\{\xi(\theta) ; \theta \in \Theta\}$ denotes the range of $\xi(\cdot)$. Clearly, $\mu^{\xi} \neq \mu$ for any choice of the map $\xi(\cdot)$.

Acknowledgment. The authors wish to thank the anonymous referees for carefully reading the manuscript and suggesting several improvements.

\section{REFERENCES}

[1] L. Ambrosio, N. Fusco, and D. Pallara, Functions of Bounded Variation and Free Discontinuity Problems, Oxford University Press, New York, 2000.

[2] D. H. Armitage and S. J. Gardiner, Classical Potential Theory, Springer-Verlag, London, 2001.

[3] P. Benilan, M. G. Crandall, and P. Sacks, Some $\mathbf{L}^{1}$ existence and dependence results for semilinear elliptic equations under nonlinear boundary conditions, Appl. Math. Optim., 17 (1998), pp. 203-224.

[4] M. Bernot, V. Caselles, and J. M. Morel, Optimal Transportation Networks. Models and Theory, Lecture Notes in Math. 1955, Springer-Verlag, Berlin, 2009.

[5] L. Boccardo and T. Gallouët, Nonlinear elliptic and parabolic equations involving measure data, J. Funct. Anal., 87 (1989), pp. 149-169.

[6] L. Boccardo, T. GallouËT, And L. Orsina, Existence and uniqueness of entropy solutions for nonlinear elliptic equations with measure data, Ann. Inst. H. Poincaré Anal. Non Linéaire, 13 (1996), pp. 539-551.

Copyright (C) by SIAM. Unauthorized reproduction of this article is prohibited. 
[7] A. Bressan, G. Coclite, And W. Shen, A multi-dimensional optimal harvesting problem with measure valued solutions, SIAM J. Control Optim., 51 (2013), pp. 1186-1202, https://doi.org/10.1137/110853510.

[8] A. Bressan And K. Han, Nash equilibria for a model of traffic flow with several groups of drivers, ESAIM Control, Optim. Calc. Var., 18 (2012), pp. 969-986.

[9] H. BREZIS AND D. KinderlehreR, The smoothness of solutions to nonlinear variational inequalities, Indiana Univ. Math. J., 23 (1974), pp. 831-844.

[10] G. Buttazzo and G. Dal Maso, Shape optimization for Dirichlet problems: Relaxed formulation and optimality conditions, Appl. Math. Optim., 23 (1991), pp. 17-49.

[11] A. Cañada, J. L. Gámez, and J. A. Montero, Study of an optimal control problem for diffusive nonlinear elliptic equations of logistic type, SIAM J. Control Optim., 36 (1998), pp. 1171-1189, https://doi.org/10.1137/S0363012995293323.

[12] G. Dal Maso, F. Murat, L. Orsina, and A. Prignet, Renormalized solutions of elliptic equations with general measure data, Ann. Scuola Norm. Sup. Pisa Cl. Sci. (4), 28 (1999), pp. 741-808.

[13] K. Deimling, Nonlinear Functional Analysis, Springer-Verlag, Berlin, 1985.

[14] J. Dieudonné, Foundations of Modern Analysis, 4th ed., Academic Press, New York, 1963.

[15] W. Ding and S. Lenhart, Optimal harvesting of a spatially explicit fishery model, Nat. Resour. Model., 22 (2009), pp. 173-211.

[16] L. C. Evans, Partial Differential Equations, Grad. Stud. Math. 19, 2nd ed., AMS, Providence, RI, 2010.

[17] L. C. Evans and R. F. Gariepy, Measure Theory and Fine Properties of Functions, CRC Press, Boca Raton, FL, 1991.

[18] H. FEDERER AND W. Ziemer, The Lebesgue set of a function whose distribution derivatives are $p$-th power summable, Indiana Univ. Math. J., 22 (1972), pp. 139-158.

[19] J. FreHSE, On the regularity of the solution of a second order variational inequality, Boll. Un. Mat. Ital. (4), 6 (1972), pp. 312-315.

[20] T. L. Friesz, Dynamic Optimization and Differential Games, Springer, Boston, 2010.

[21] G. Hardin, The tragedy of the commons, Science, 162 (1968), pp. 1243-1248.

$[22]$ G. E. Herrera And S. Lenhart, Spatial optimal control of renewable resource stocks, in Spatial Ecology, Mathematical and Computational Biology Series, Chapman \& Hall, CRC, Boca Raton, FL, 2010, pp. 343-357.

[23] M. Kelly, Y. Xing, and S. Lenhart, Optimal fish harvesting for a population modeled by a nonlinear parabolic differential equation, Nat. Resour. Model., 29 (2016), pp. 36-70.

[24] D. Kinderlehrer and G. Stampacchia, An Introduction to Variational Inequalities and Their Applications, Academic Press, New York, London, 1980.

[25] M. Neubert, Marine reserves and optimal harvesting, Ecol. Lett., 6 (2003), pp. 843-849.

[26] J. F. Rodrigues, Obstacle Problems in Mathematical Physics, North-Holland, Amsterdam, 1987.

Copyright $@$ by SIAM. Unauthorized reproduction of this article is prohibited. 